ANALYSIS \& PDE Volume $6 \quad$ No. $8 \quad 2013$

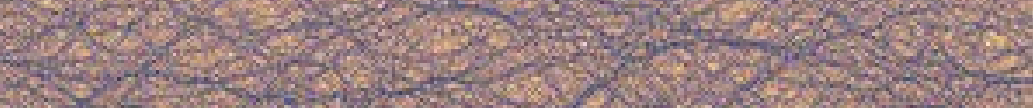
NOAH SNYDER

A ROTATIONAL APPROACH TO TRIPLE POINT OBSTRUCTIONS 


\title{
A ROTATIONAL APPROACH TO TRIPLE POINT OBSTRUCTIONS
}

\author{
NOAH SNYDER
}

\begin{abstract}
Subfactors where the initial branching point of the principal graph is 3-valent are subject to strong constraints called triple point obstructions. Since more complicated initial branches increase the index of the subfactor, triple point obstructions play a key role in the classification of small index subfactors. There are two strong triple point obstructions, called the triple-single obstruction and the quadratic tangles obstruction. Although these obstructions are very closely related, neither is strictly stronger. In this paper we give a more general triple point obstruction which subsumes both. The techniques are a mix of planar algebraic and connection-theoretic techniques with the key role played by the rotation operator.
\end{abstract}

\section{Introduction}

The principal graph of a subfactor begins with a type $A$ string and then hits an initial branch point (unless the graph is $A_{k}$ or $A_{\infty}$ ). It is natural to stratify subfactors based on how complex this initial branch point is. Furthermore, complex initial branches increase the norm of the graph and thus the index of the subfactor. This means that small index subfactors can only have simple initial branches. The simplest possibility is an initial triple point (in this case the dual graph also begins with a triple point). Subfactors beginning with an initial triple point are subject to strong constraints known as triple point obstructions. For example, a triple point obstruction due to Ocneanu shows that as long as the index is greater than 4 the initial triple point must be at odd depth. These triple point obstructions play a crucial role in the classification of small index subfactors [Haagerup 1994; Morrison and Snyder 2012; Morrison et al. 2012; Izumi et al. 2012; Penneys and Tener 2012].

The current state of the art of triple point obstructions is given in our joint paper with S. Morrison, D. Penneys, and E. Peters [Morrison et al. 2012], but the status is somewhat unsatisfactory as there are two main results, neither of which is strictly stronger than the other. One result applies more generally and proves a certain inequality, while the other (due to V. F. R. Jones [2012]) has stricter assumptions but replaces the inequality with a finite list of values. The former is proved using connections and the latter using planar algebras. The main result of this paper is a mutual generalization of these two triple point obstructions, which proves the stronger conclusion using only the weaker assumptions. As one might expect, this paper uses a mix of connections and planar algebras following our earlier paper with M. Izumi, Jones, and Morrison [Izumi et al. 2012]. Furthermore, one can think of this argument as giving an alternate proof of the triple point obstruction from [Jones 2012].

MSC2010: 46L37.

Keywords: subfactors, planar algebras, connections. 
Before stating the three relevant results, we fix some notation which we will use throughout the paper. Suppose that $N \subset M$ is an $n-1$ supertransitive finite index subfactor of index greater than 4 whose principal graphs begin with triple points, and let $\Gamma$ and $\Gamma^{\prime}$ denote the principal and dual principal graphs. Let $[k]$ denote the quantum number $\left(v^{k}-v^{-k}\right) /\left(v-v^{-1}\right)$, where $v$ is a number such that the index is $[2]^{2}$. Let $\beta$ and $\beta^{\prime}$ denote the initial triple points at depth $n-1$ (which is necessarily odd by Ocneanu's obstruction), let $\alpha_{1}$ and $\gamma_{1}$ be the vertices at depth $n-2$, let $\alpha_{2}$ and $\alpha_{3}$ be the two vertices at depth $n$ on $\Gamma$, and let $\gamma_{2}$ and $\gamma_{3}$ be the two vertices at depth $n$ on $\Gamma^{\prime}$. We will conflate vertices with the corresponding simple bimodules and the corresponding simple projections in the planar algebra. Assume without loss of generality that $\operatorname{dim} \alpha_{2} \geq \operatorname{dim} \alpha_{3}$ and $\operatorname{dim} \gamma_{2} \geq \operatorname{dim} \gamma_{3}$.

Theorem 1 (triple-single obstruction [Morrison et al. 2012, Theorem 3.5]). If $\gamma_{3}$ is 1-valent, then

$$
\operatorname{dim}\left(\alpha_{2}\right)-\operatorname{dim}\left(\alpha_{3}\right) \leq 1 .
$$

Theorem 2 (quadratic tangles obstruction [Jones 2012]). Suppose that $\gamma_{3}$ is 1-valent and that $\gamma_{2}$ is 3-valent; then

$$
r+\frac{1}{r}=\frac{\lambda+\lambda^{-1}+2}{[n][n+2]}+2,
$$

where $\lambda$ is the scalar by which rotation acts on the 1-dimensional perpendicular complement of TemperleyLieb at depth $n$ and $r=\operatorname{dim}\left(\alpha_{2}\right) / \operatorname{dim}\left(\alpha_{3}\right)$.

Since $\lambda$ is a root of unity, we know that $-2 \leq \lambda+\lambda^{-1} \leq 2$. Hence the quadratic tangles obstruction gives an inequality, and (as observed by Zhengwei Liu) this inequality turns out to be precisely the one in the triple-single obstruction [Morrison et al. 2012, Lemma 3.3]. Thus the quadratic tangles obstruction is stronger (replacing an interval of possibilities with a finite list) when both apply, but the triple-single obstruction has a weaker assumption. The main result of this paper is the following mutual generalization of Theorems 1 and 2.

Theorem 3. Suppose that $\gamma_{3}$ is 1-valent; then

$$
r+\frac{1}{r}=\frac{\lambda+\lambda^{-1}+2}{[n][n+2]}+2 .
$$

\section{Background}

We quickly summarize the key idea of [Izumi et al. 2012, §5.2], which is that the action of rotation on the planar algebra can be read off from the connection. Since rotational eigenvalues must be roots of unity, this gives highly nontrivial constraints on candidate connections. We assume that the reader is familiar with both planar algebras and connections; see [Izumi et al. 2012] for more detail.

Given a subfactor $N \subset M$ we get a certain collection of matrices called a connection. This connection depends on a choice of certain intertwiners, and thus is only well-defined up to gauge automorphisms. Let the branch matrix $U$ denote the 3-by-3 matrix coming from the connection at the initial branch vertex of $\Gamma$. The key idea of Izumi et al. is that there is a canonical gauge choice for $U$, called the diagrammatic branch 
matrix, coming from the planar algebra. This choice is both easy to recognize and has nice properties, as captured by the following two results.

Lemma 4 [Izumi et al. 2012, Lemma 5.6]. When $n$ is odd the diagrammatic branch matrix is characterized within its gauge class by the property that all the entries in the first row and column are positive real numbers.

Proposition 5. Let $U$ be the diagrammatic branch matrix for a subfactor with an initial triple point. Let $x$ be an $n$-box in the perpendicular complement of Temperley-Lieb, and write $x=a_{2}\left(\alpha_{2} / \sqrt{\operatorname{dim} \alpha_{2}}\right)+$ $a_{3}\left(\alpha_{3} / \sqrt{\operatorname{dim} \alpha_{3}}\right)$. Let $\left(c_{1}, c_{2}, c_{3}\right)=U\left(0, a_{2}, a_{3}\right)$. Then $c_{1}=0$ and $c_{2}\left(\gamma_{2} / \sqrt{\operatorname{dim} \gamma_{2}}\right)+c_{3}\left(\gamma_{3} / \sqrt{\operatorname{dim} \gamma_{3}}\right)$ is $\rho^{1 / 2}(x)$.

Proof. This is a restatement of [Izumi et al. 2012, Corollary 5.3] in our special case. See [Izumi et al. 2012, pp. 18-19] for a worked example.

In order to apply the previous proposition, we will want an explicit formula for vectors in the perpendicular complement to Temperley-Lieb in the $n$-box space and the action of rotation there. Recall that the rotation $\rho$ preserves shading and thus is an endomorphism of each box space, while $\rho^{1 / 2}$ changes rotation and thus is a map from one box space to a different box space. We will use $\lambda$ to denote the scalar by which $\rho$ acts on the 1-dimensional perpendicular complement to Temperley-Lieb in the $n$-box space. Note that this is an $n$-th root of unity.

Lemma 6. Let $r=\operatorname{dim} \alpha_{2} / \operatorname{dim} \alpha_{3}$ and $\check{r}=\operatorname{dim} \gamma_{2} / \operatorname{dim} \gamma_{3}$. Then $T=(1 / \sqrt{r}) \alpha_{2}-\sqrt{r} \alpha_{3}$ and $\check{T}=$ $(1 / \sqrt{\breve{r}}) \gamma_{2}-\sqrt{\check{r}} \gamma_{3}$ are each in the perpendicular complement of Temperley-Lieb.

Furthermore $\rho^{1 / 2}(T)=\sqrt{\lambda} \breve{T}$, where $\sqrt{\lambda}$ is some square root of the rotational eigenvalue for the action of rotation on the perpendicular compliment of Temperley-Lieb.

Proof. These calculations (with slightly different conventions) were done in an early version of [Jones 2012]. Seeing that $T$ and $\check{T}$ are perpendicular to Temperley-Lieb is straightforward (you only need to work out their inner product with two specific Jones-Wenzl projections). Since half-click rotation preserves Temperley-Lieb and is an isometry, it also preserves the perpendicular complement of Temperley-Lieb. Thus $\rho^{1 / 2}(T)$ is some scalar multiple of $\check{T}$. To work out which scalar multiple this is you compute their norms. This tells you that the square of this scalar is $\lambda$.

Remark 7. There are many square roots in this paper. Other than $\sqrt{\lambda}$, all square roots are positive square roots of positive numbers. Moreover $\sqrt{\lambda}$ will always be chosen such that the previous lemma works. In the final statement of the main theorem no $\sqrt{\lambda}$ appears, so this subtlety is not very important.

Combining the previous two results we have the following concrete statement, which will supply the main ingredient of our proof of Theorem 3.

Corollary 8. The diagrammatic branch matrix $U$ sends

$$
\left(0, \sqrt{\operatorname{dim}\left(\alpha_{3}\right)},-\sqrt{\operatorname{dim}\left(\alpha_{2}\right)}\right) \mapsto \sqrt{\lambda}\left(0, \sqrt{\operatorname{dim}\left(\gamma_{3}\right)},-\sqrt{\operatorname{dim}\left(\gamma_{2}\right)}\right) .
$$




\section{Proof of Theorem 3}

The idea of this argument is that having a 1-valent vertex allows us to solve for the branch matrix, and thus we can read off the rotational eigenvalue (since the diagrammatic branch matrix acts on the appropriate vectors by rotation). This gives an identity between the dimensions of objects and the rotational eigenvalue.

We begin with a quick calculation of the branch matrix following the proof of the triple-single obstruction [Morrison et al. 2012, Theorem 3.1]. Since $\alpha_{1}, \gamma_{1}, \beta$, and $\beta^{\prime}$ are in the initial string their dimensions are $[n-1],[n-1],[n]$, and $[n]$, respectively. Since $\gamma_{3}$ is 1 -valent, we have $\operatorname{dim} \gamma_{2}=[n+2] /[2]$ and $\operatorname{dim} \gamma_{3}=[n] /[2]$. Using the 1-valence of $\gamma_{3}$ the normalization condition on connections determines the magnitude of several of the entries in the branch matrix. Furthermore, unitarity of $U$ allows us to work out several more of the entries. In particular, the branch matrix is gauge equivalent to the matrix below, where $p=\operatorname{dim}\left(\alpha_{2}\right)$ and $q=\operatorname{dim}\left(\alpha_{3}\right)$, where $\sigma$ and $\tau$ are unknown phases, and where ? denotes unknown entries which will play no role in the calculation.

$$
U=\left(\begin{array}{ccc}
\frac{1}{[n]} & \frac{\sqrt{[n-1] p}}{[n]} & \frac{\sqrt{[n-1] q}}{[n]} \\
\sqrt{\frac{[n-1]}{[2][n]}} & \sigma \sqrt{\frac{p}{[2][n]}} & \tau \sqrt{\frac{q}{[2][n]}} \\
\sqrt{\frac{[n-1][n+2]}{[2][n]^{2}}} & ? & ?
\end{array}\right) .
$$

The first row and column of this matrix are clearly positive, so by Lemma 4 we see that $U$ is the diagrammatic branch matrix.

Remark 9. This matrix is the transpose of the matrix found in [Morrison et al. 2012] because the calculation there is done for $\Gamma^{\prime}$ instead of $\Gamma$. As shown in [Izumi et al. 2012], the diagrammatic branch matrices of $\Gamma$ and $\Gamma^{\prime}$ are always transposes.

We would like to solve for $\sigma$ and $\tau$. Orthogonality of the first two rows of $U$ tells us that

$$
1+\sigma p+\tau q=0 .
$$

Although $1+\sigma p+\tau q=0$ is one equation in two unknowns, it actually determines $\sigma$ and $\tau$ since they are phases:

$$
\begin{gathered}
\sigma=-\frac{1+\tau q}{p}, \\
1=\sigma \bar{\sigma}=\frac{1+\tau q}{p} \frac{1+\bar{\tau} q}{p}=\frac{1+(\tau+\bar{\tau}) q+q^{2}}{p^{2}}, \\
\tau+\bar{\tau}=\frac{p^{2}-q^{2}-1}{q} .
\end{gathered}
$$

This determines the real part of $\tau$, and thus $\tau$ itself. Similarly, $\sigma+\bar{\sigma}=\left(q^{2}-p^{2}-1\right) / p$. 
Now that we have a very explicit understanding of $U$ we apply it to a rotational eigenvector. Corollary 8 tells us that $U$ sends

$$
(0, \sqrt{q},-\sqrt{p}) \mapsto \sqrt{\lambda}\left(0, \sqrt{\frac{[n+2]}{[2]}},-\sqrt{\frac{[n]}{[2]}}\right) .
$$

Looking at the middle coordinate of that identity, we see that

$$
\sigma-\tau=\sqrt{\lambda} \sqrt{\frac{[n+2][n]}{p q}} .
$$

Comparing the real parts of both sides yields

$$
\begin{aligned}
\left(\sqrt{\lambda}+\frac{1}{\sqrt{\lambda}}\right) \sqrt{\frac{[n+2][n]}{p q}} & =(\sigma+\bar{\sigma})-(\tau+\bar{\tau}) \\
& =\frac{q^{2}-p^{2}-1}{p}-\frac{p^{2}-q^{2}-1}{q}=\frac{(q-p)\left((p+q)^{2}-1\right)}{p q} \\
& =\frac{(q-p)\left([n+1]^{2}-1\right)}{p q}=\frac{(q-p)([n][n+2])}{p q} .
\end{aligned}
$$

Squaring both sides and rearranging proves the theorem.

Remark 10. You might guess that $\sigma-\tau=\sqrt{\lambda} \sqrt{[n+2][n] /(p q)}$ would give a second condition coming from the imaginary parts. In fact there's no new information there, because the two sides automatically have the same norm.

\section{Acknowledgements}

The main ideas in this paper came out of joint work with Scott Morrison, and I would like to thank him for many helpful conversations. I would also like to thank Vaughan Jones, Dave Penneys, and Emily Peters. This work was supported by an NSF Postdoctoral Fellowship at Columbia University and DARPA grants HR0011-11-1-0001 and HR0011-12-1-0009.

\section{References}

[Haagerup 1994] U. Haagerup, "Principal graphs of subfactors in the index range $4<[M: N]<3+\sqrt{2}$ ", pp. 1-38 in Subfactors (Kyuzeso, 1993), edited by H. Araki et al., World Scientific, River Edge, NJ, 1994. MR 96d:46081 Zbl 0933.46058

[Izumi et al. 2012] M. Izumi, V. F. R. Jones, S. Morrison, and N. Snyder, "Subfactors of index less than 5, Part 3: Quadruple points", Comm. Math. Phys. 316:2 (2012), 531-554. MR 2993924 Zbl 1272.46051 arXiv 1109.3190

[Jones 2012] V. F. R. Jones, “Quadratic tangles in planar algebras”, Duke Math. J. 161:12 (2012), 2257-2295. MR 2972458 Zbl 1257.46033 arXiv 1007.1158

[Morrison and Snyder 2012] S. Morrison and N. Snyder, "Subfactors of index less than 5, part 1: The principal graph odometer", Comm. Math. Phys. 312:1 (2012), 1-35. MR 2914056 Zbl 1246.46055 arXiv 1007.1730

[Morrison et al. 2012] S. Morrison, D. Penneys, E. Peters, and N. Snyder, "Subfactors of index less than 5, Part 2: Triple points", Internat. J. Math. 23:3 (2012), 1250016, 33. MR 2902285 Zbl 1246.46054 arXiv 1007.2240

[Penneys and Tener 2012] D. Penneys and J. E. Tener, "Subfactors of index less than 5, part 4: Vines", Internat. J. Math. 23:3 (2012), 1250017, 18. MR 2902286 Zbl 1246.46056 
Received 3 Oct 2012. Revised 22 Jan 2013. Accepted 8 Mar 2013.

NOAH SNYDER: nsnyder@math.indiana.edu

Mathematics Department, Indiana University, 831 E. Third St., Bloomington, IN 47401, United States 


\title{
Analysis \& PDE
}

\author{
msp.org/apde
}

\section{EDITORS}

EDITOR-IN-CHIEF

\author{
Maciej Zworski \\ zworski@math.berkeley.edu \\ University of California \\ Berkeley, USA
}

BOARD OF EDITORS

Nicolas Burq Université Paris-Sud 11, France

nicolas.burq@math.u-psud.fr

Sun-Yung Alice Chang Princeton University, USA

chang@math.princeton.edu

Michael Christ University of California, Berkeley, USA

mchrist@math.berkeley.edu

Charles Fefferman Princeton University, USA

cf@math.princeton.edu

Ursula Hamenstaedt Universität Bonn, Germany

ursula@math.uni-bonn.de

Vaughan Jones U.C. Berkeley \& Vanderbilt University vaughan.f.jones@vanderbilt.edu

Herbert Koch Universität Bonn, Germany koch@math.uni-bonn.de

Izabella Laba University of British Columbia, Canada ilaba@math.ubc.ca

Gilles Lebeau Université de Nice Sophia Antipolis, France lebeau@unice.fr

László Lempert Purdue University, USA lempert@math.purdue.edu

Richard B. Melrose Massachussets Institute of Technology, USA rbm@math.mit.edu

Frank Merle Université de Cergy-Pontoise, France Frank.Merle@u-cergy.fr

William Minicozzi II Johns Hopkins University, USA minicozz@math.jhu.edu

Werner Müller Universität Bonn, Germany mueller@math.uni-bonn.de
Yuval Peres

Gilles Pisier

Tristan Rivière

Igor Rodnianski

Wilhelm Schlag

Sylvia Serfaty

Yum-Tong Siu

Terence Tao

Michael E. Taylor

Gunther Uhlmann

András Vasy

Dan Virgil Voiculescu

Steven Zelditch
University of California, Berkeley, USA

peres@stat.berkeley.edu

Texas A\&M University, and Paris 6

pisier@math.tamu.edu

ETH, Switzerland

riviere@math.ethz.ch

Princeton University, USA

irod@math.princeton.edu

University of Chicago, USA

schlag@math.uchicago.edu

New York University, USA

serfaty@cims.nyu.edu

Harvard University, USA

siu@math.harvard.edu

University of California, Los Angeles, USA

tao@math.ucla.edu

Univ. of North Carolina, Chapel Hill, USA

met@math.unc.edu

University of Washington, USA

gunther@math.washington.edu

Stanford University, USA

andras@math.stanford.edu

University of California, Berkeley, USA

dvv@math.berkeley.edu

Northwestern University, USA

zelditch@math.northwestern.edu

\section{PRODUCTION}

production@msp.org

Silvio Levy, Scientific Editor

See inside back cover or msp.org/apde for submission instructions.

The subscription price for 2013 is US \$160/year for the electronic version, and \$310/year ( $\$ 35$, if shipping outside the US) for print and electronic. Subscriptions, requests for back issues from the last three years and changes of subscribers address should be sent to MSP.

Analysis \& PDE (ISSN 1948-206X electronic, 2157-5045 printed) at Mathematical Sciences Publishers, 798 Evans Hall \#3840, c/o University of California, Berkeley, CA 94720-3840, is published continuously online. Periodical rate postage paid at Berkeley, CA 94704, and additional mailing offices.

APDE peer review and production are managed by EditFLOW ${ }^{\circledR}$ from Mathematical Sciences Publishers.

PUBLISHED BY

- mathematical sciences publishers

nonprofit scientific publishing

http://msp.org/

(C) 2013 Mathematical Sciences Publishers 


\section{ANALYSIS \& PDE \\ Volume $6 \quad$ No. $8 \quad 2013$}

$L^{p}$ and Schauder estimates for nonvariational operators structured on Hörmander vector fields 1793 with drift

Marco Bramanti and MaOchun ZhU

Strichartz estimates for Schrödinger equations with variable coefficients and unbounded po- 1857 tentials

HARUYA MIZUTANI

Uniformity of harmonic map heat flow at infinite time

1899

LONGZHI LIN

A rotational approach to triple point obstructions

1923

NOAH SNYDER

On the energy subcritical, nonlinear wave equation in $\mathbb{R}^{3}$ with radial data RUIPENG SHEN

Global well-posedness for the nonlinear Schrödinger equation with derivative in energy space 1989 YIFEI WU

The Calderón problem with partial data on manifolds and applications

2003

CARlos Kenig and MikKo SAlo 